ISSN: 0514-7336

DOI: http://dx.doi.org/10.14201/zephyrus201576195199

\title{
NUEVO EPÍGRAFE ROMANO PROCEDENTE DEL CORTINAL DE SAN JUAN (SALVATIERRA DE TORMES, SALAMANCA)
}

\section{An unpublished Roman inscription from Cortinal de San Juan (Salvatierra de Tormes, Salamanca)}

\author{
Verónica PÉRez De Dios
}

Dpto. de Prehistoria, Historia Antigua y Arqueología. Facultad de Geografía e Historia. C/ Cervantes, s/n. 37002 Salamanca. Correo-e: veropdd@usal.es

Recepción: 25/01/2015; Revisión: 6/07/2015; Aceptación: 14/09/2015

BIBLID [0514-7336 (2015) LXXVI, julio-diciembre; 195-199]

\begin{abstract}
Resumen: En este artículo se presenta una pizarra con una inscripción de época romana hallada en los trabajos de prospección arqueológica llevados a cabo en septiembre de 2014 en el Cortinal de San Juan (Salvatierra de Tormes). Este yacimiento ha sido, desde su descubrimiento y catalogación, un enclave de referencia para el poblamiento tardorromano y visigodo del SE de la provincia de Salamanca y, actualmente, se encuentra parcialmente inundado por el embalse de Santa Teresa. La principal particularidad de este epígrafe radica en que el uso de la pizarra como soporte no es habitual en la cronología propuesta para dicha inscripción. A pesar de que el resto de los materiales arqueológicos encontrados en los trabajos de prospección del Cortinal de San Juan están asociados a un contexto cronocultural tardío o visigodo, la tipología de letra capital del epígrafe, el gran tamaño de esta y los paralelos establecidos con otros hallazgos de datación similar hacen cobrar fuerza a la hipótesis interpretativa que argumenta que dicha inscripción podría formar parte de un antropónimo de origen romano.

Palabras clave: Pizarra; inscripción romana; antropónimo; necrópolis; sureste de Salamanca; poblamiento rural.
\end{abstract}

Aвsтract: In this paper is presented a slate with a Roman inscription found in archaeological survey work conducted in September 2014 in Cortinal de San Juan (Salvatierra de Tormes). This site has been, since its discovery and cataloging, a place of reference for the late Roman and Visigothic settlement Southeast Salamanca. Currently, this archaeological site is partially flooded by the Santa Teresa swamp. The main feature of this Roman inscription is that the slate support isn't usual in the chronology proposed for such inscription. Despite the fact that other archaeological materials of Cortinal de San Juan are associated with late Roman or Visigothic chronocultural context, the type of capital letter, the large size of the letter, and the parallels established with other finds of similar chronology suggest this inscription could be part of a Roman anthroponym as the most successful interpretive hypothesis.

Key words: Slates; Roman inscription; anthroponym; cemetery; Southeast of Salamanca; rural settlement.

(C) Universidad de Salamanca

Zephyrus, LXXVI, julio-diciembre 2015, 195-199 


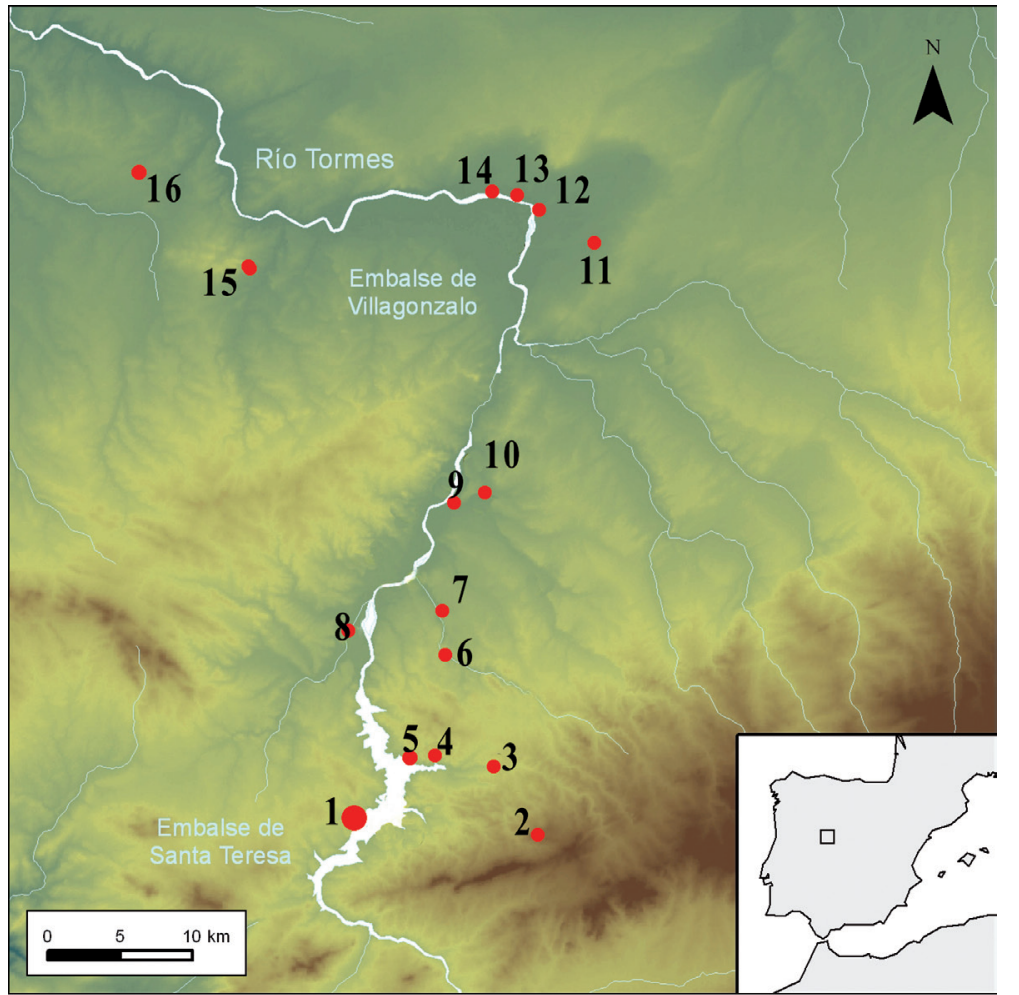

1- Cortinal de San Juan

2- Las Quintanas

3- Pedro Fuertes

4- El Colmenar

5- Dehesa del Cañal

6- Martin Perez

7- La Vega de Santa Ines

8- La Maya
9- Portillo

10-Revilla la Baja

11-San Vicente del rio Almar

12- Los Bebederos

13- Aceña de la Fuente

14- San Pelayo

15-MIranda de Azan

16- Galindo y Perahuy

Fig. 1. Mapa de distribución de yacimientos con hallazgos de pizarras.

\section{Introducción ${ }^{1}$}

En la comarca de Salvatierra de Tormes, las primeras referencias a evidencias arqueológicas fueron publicadas a principios del s. Xx por el padre C. Morán (1919: 112; [1946] 2000) y M. Gómez Moreno (1967: 81), quienes sacaron a la luz las primeras pizarras numerales adscritas a cronología visigoda encontradas en el yacimiento del Cortinal de San Juan. A mediados del s. xx, J. Maluquer (1956: 104-105),

1 Este trabajo está enmarcado en el proyecto de tesis doctoral sobre Poblamiento romano y altomedieval del valle medio del Tormes que estamos desarrollando con una ayuda económica cofinanciada por la Univ. Salamanca y el Banco de Santander (2015-2019). en su Carta Arqueológica de España: Salamanca, resaltó la aparición de dos restos mucho más trascendentes: un cancel de mármol del s. VII con bajorrelieve de crismones y pavos reales y la existencia de una necrópolis tardorromana en el yacimiento del Regato de la Silla II. Posteriormente, en 1973, y debido a la importancia de los hallazgos casuales, E. Cerrillo realizó excavaciones arqueológicas en el Cortinal de San Juan que, además de dar a conocer 33 nuevas pizarras y una moneda de Magno Máximo, pusieron de manifiesto que el enclave se extendía hacia el so del elevado cerro en el que se localiza el yacimiento (Cerrillo, 1977: 313-318; Ariño, 2011: 255). Desde las excavaciones realizadas por Cerrillo, únicamente se han realizado cuatro campańas de prospección por parte de M. Santonja (1984), L. Sanz de M. ARQUEOTIPO S.C.L. (1998-1999), Gorbea Pérez de M. GRupoentorno S.A. (2004-2005) y V. Pérez de Dios (2013-2014); en ellas se han localizado cuantiosos fragmentos de material constructivo -teja, ladrillo, fustes de columnas y grandes sillares de granito- y material cerámico -cerámica común de cocina, cerámica común, paredes finas, cerámica estampillada o imitación de sigillatas, sigillata hispánica, sigillata hispánica tardía y dolia-; también se han recuperado numerosos fragmentos de molino, varios elementos metálicos entre los que cabe destacar una moneda de Teodosio (379-395) y algunas pizarras numerales ${ }^{2}$.

\section{Localización del Cortinal de San Juan}

Emplazado en un espigón del río Tormes, se caracteriza por presentar un relieve suavemente ondulado en el que existen grandes afloramientos de pizarra

2 Para más detalles sobre estos materiales en Pérez de Dios, V.: El poblamiento romano y tardoantiguo en el Sureste de la provincia de Salamanca, Trabajo de Fin de Máster inédito presentado en 2014 en la UNED, pp. 139-150. 
(Dorronsoro, 1991: 478-506) y no se presenta en el paisaje salmantino como un yacimiento aislado, sino que forma parte de un contexto más amplio de asentamientos adscritos a cronologías tardorromanas y visigodas localizados en el valle medio del río Tormes (Ariño, 2011: 251-270; Díaz Martínez y Martín Viso, 2011: 221-250).

Por su situación geográfica, el enclave debió gozar de una economía próspera ligada a la abundancia de tierras muy fértiles, hasta los años 60 del siglo pasado, momento en el que se produjo la construcción del embalse de Santa Teresa. El levantamiento de esta nueva presa provocó un incremento del caudal del río Tormes que desencadenó la inundación completa o parcial de varios yacimientos del área de influencia de Salvatierra de Tormes y del puente, de dudosa cronología romana o medieval, que unía Salvatierra de Tormes con las poblaciones de La Tala, Pelayos y Galinduste ${ }^{3}$.

\section{El nuevo epígrafe del Cortinal de San Juan}

\subsection{Descripción física}

El soporte epigráfico corresponde a una placa de pizarra grisácea, con algunas concreciones rojizas en la parte inferior, que presenta una superficie escamosa. La placa de pizarra tiene forma alargada y grandes dimensiones: $29,5 \mathrm{~cm}$ x $61 \mathrm{~cm}$, un grosor de $6,5 / 4 \mathrm{~cm}$ y $22 \mathrm{~kg}$ de peso, mostrando una única fractura en su lateral izquierdo que afecta parcialmente a la lectura del texto. Los caracteres parecen corresponder a la parte final del epígrafe pues la pizarra no presenta fractura en la parte derecha, área donde el reborde aparece pulido. Superficialmente no se observa una preparación previa de la placa previa a la inscripción, como el pulido de la cara anterior; por ello, lo más factible es que el soporte epigráfico fuera seleccionado por su desmesurada envergadura. La pieza está exclusivamente grabada en su cara superior, en la que se

$3 \quad$ Ibidem, p. 139.
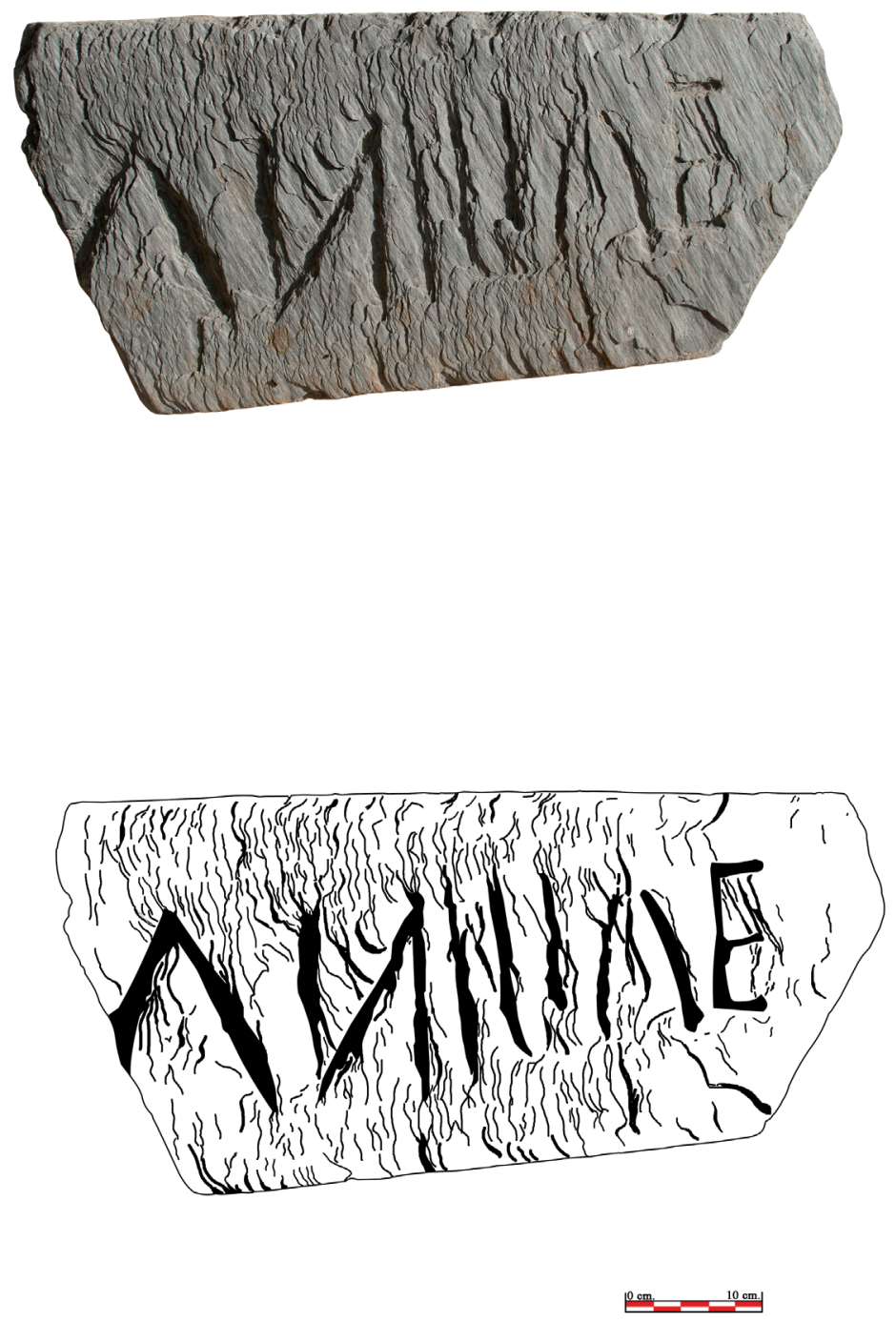

Fig. 2. Fotografía y dibujo del epigrafe hallado en el Cortinal de San Juan. conservan siete caracteres de diversos tamaños labrados con un cincel grueso y metálico. El reverso no presenta ninguna modificación antrópica.

Todos los caracteres representados en la pizarra son letras capitales alargadas que comienzan a utilizarse en Hispania a lo largo del s. III y que fueron labradas mediante la realización de trazos largos y rectos (Andreu, 2009: 103). La ordinatio aparentemente es descuidada, pues se aprecian inconsistencias en la longitud de las letras del epígrafe cuya altura varía desde los $15 \mathrm{~cm}$ de la primera A hasta los $12 \mathrm{~cm}$ de la 
E final. Además de esta oscilación longitudinal de 3 $\mathrm{cm}$, el grosor de los caracteres también fluctúa entre $\operatorname{los} 2 / 1,5 \mathrm{~cm}$.

\subsection{Conservación y lectura}

El estado de conservación del epígrafe del Cortinal del San Juan es bueno pues, a pesar de estar en contacto directo con la acción erosiva del agua, no presenta una exfoliación notable en la capa superficial del soporte epigráfico. Por ello es posible proponer la siguiente lectura para la inscripción: [---] ANILIAE [---].

En primer lugar, observamos un carácter que, aunque se presenta incompleto, puede identificarse con la letra A. Esta A inicial se caracteriza, al igual que la penúltima letra del epígrafe, por presentar únicamente dos trazos oblicuos y carecer de trazo transversal, siendo así su grafía idéntica a la de una $\mathrm{v}$ invertida. La segunda letra que conforma el epígrafe parece identificarse con el carácter $\mathrm{N}$, aunque su representación responde a una forma muy singular pues el trazo medio aparece representado a la inversa, es decir, uniendo el lado inferior del primer trazo con el superior del segundo. Además de estos dos caracteres, podemos señalar que el último trazo del epígrafe corresponde a la letra E. Dicha grafía está formada por cuatro trazos, siendo los tres horizontales muy regulares en longitud y grosor.

Además de las cuatro letras identificadas con exactitud, el epígrafe está formado por otros tres caracteres epigráficos de trazo recto y vertical de difícil interpretación. Dichos caracteres pueden representar varias letras del alfabeto latino ya que pueden ser entendidas como I, L o T, aunque también es posible que dos de los trazos representaran una $\mathrm{E}$, carácter habitual en las inscripciones en las que se utiliza el latín arcaico.

\subsection{Interpretación textual}

Tras el análisis de los elementos externos del epígrafe se ha procedido a la realización de un estudio intrínseco del significado del texto. En este sentido, el principal problema de la inscripción reside en la pérdida de los primeros caracteres epigráficos que conforman el cognomen del difunto, lo que dificulta notablemente su comprensión. Por contraposición, el final del epígrafe es la parte que presenta una lectura más clara porque la terminación - $\mathrm{AE}-$ es frecuentemente utilizada en la lengua latina. En base a esto, se han propuesto varias opciones de lectura, siendo la más factible la hipótesis que sugiere que el epígrafe fuera un antropónimo escrito en nominativo, genitivo o dativo; debido a la ausencia de una línea superior en el epígrafe falta la previa y habitual invocación a los Dioses Manes. En este sentido, se han podido establecer algunos paralelos con otras inscripciones de Hispania que presentan caracteres epigráficos muy similares en los que el cognomen aparece completo (Abascal, 1994 y Kajanto, 1965): ANELIA en Valdenebro de los Valles, Valladolid (Palol, 1964: 307-311; García y Bellido, 1966: 395-396; Mañanes y Solana, 1999: 41-42), y ANILIA en Alcaudete, Jaén (AEp, 1992, 1072). Además de estas dos opciones, se contempla la posibilidad de que el epígrafe del Cortinal de San Juan no estuviera completo. De ser así, el cognomen podría tener diversas variantes de las que se tiene constancia en los corpora epigráficos: CANILLA, cuyo paralelo puede establecerse con la inscripción de Tarraco en la que se nombra a CLODIa CANILla (CIL II, 6108); hispanilla, cognomen comparable al epitafio sepulcral de Talavera de la Reina en el que se nombra a ARRIA HISPANILla (ILER, 5648; Rivero, 1931: 5-6; 1933: 40); FUlvianilla, que podría asemejarse a la inscripción de Barcelona en la que puede leerse SERGIA FULVIANILLA (CIL II, 4522); SILVANILLAE, cuyo cognomen aparece documentado en una inscripción de la provincia de Lusitania (Batalha, Leiria, Portugal) en la que se puede leer CLAV [DiAE] SILVANILlaE (CIl iI, 340), y con el cognomen Firmanilla, del que tenemos constancia en una inscripción triple aparecida en la casa de los Abarca Maldonado en la propia ciudad de Salamanca (Morán, 1933: 389-391).

Teniendo en cuenta la morfología, las dimensiones de la pizarra y los posibles cognomina con los que se han podido establecer paralelos podemos argumentar que lo más previsible es que el texto formara parte de un epígrafe funerario en el que únicamente podemos distinguir parte del nombre del difunto ${ }^{4}$, sin que se haga mención a la filiación o a la edad del fallecido. A pesar de que la información contenida en el epígrafe es reducida, los estudios comparativos parecen indicar

4 Aunque no es frecuente encontrar epígrafes en los que sólo aparece el nombre del fallecido se tiene constancia de la existencia de algunos, dato que, según Calabi (1968: 201 y 338 ), permitiría suponer que habría sido encargado en vida por el difunto. 
que esta inscripción podría ser un nomen utilizado en posición de cognomen, lo que nos indicaría que habría sido utilizado como nombre de mujer.

\section{Conclusión}

La ausencia de un contexto arqueológico asociado a una estratigrafía específica impide adscribir la pizarra a una cronología concreta. Pese a ello, si primamos la relevancia de los factores internos de la grafía frente al contexto arqueológico de un yacimiento que presenta una estratigrafía y unos materiales alterados por sus continuas inundaciones, podemos asociar dicha inscripción a la ocupación cronocultural romana del asentamiento, una época en la que la pizarra no es un soporte frecuente. A pesar de ello, creemos que su elección podría haber estado condicionada por la abundancia de esta materia prima en el área del Cortinal de San Juan. Junto a la riqueza pizarrosa, el hecho de que la mayor parte de estos soportes no requieran una preparación especial, podría haber sido otro de los factores claves por los que dicho soporte habría sido considerado como el más apropiado para realizar las anotaciones cotidianas del asentamiento. Sin lugar a dudas, ambos factores contribuyeron al incremento del uso de la pizarra como soporte escriptorio en el posterior periodo de ocupación visigoda del Cortinal de San Juan (Velázquez, 1989, 2004).

Finalmente, cabe señalar que el estudio contextual, la morfología de la pizarra y el análisis de los caracteres epigráficos nos permiten concluir que, lo más probable es que el texto de esta inscripción funeraria pudo haber formado parte de un antropónimo femenino que podría estar estrechamente relacionado con la antigua necrópolis del Cortinal de San Juan citada por Maluquer (1956: 104-105) y que actualmente se encuentra bajo las aguas del embalse de Santa Teresa.

\section{Bibliografía}

Abascal, J. M (1994): Los nombres personales en las inscripciones latinas de Hispania. Murcia: Univ. de Murcia-Univ. Complutense de Madrid.

Andreu, J. (coord.) (2009): Fundamentos de Epigrafía Latina. Madrid: Liceus E-Excellence.

Ariño, E. (2011): "El yacimiento de El Cortinal de San Juan (Salvatierra de Tormes). Problemas de datación y contexto”. En Díaz Martínez, P. C. y Martín Viso, I. (eds.):
Entre el impuesto y la renta. Problemas de la fiscalidad tardoantigua y altomedieval. Bari, pp. 251-270.

Calabi, I. (1968): Epigrafía latina. Milán: Instituto Editoriale Cisalpino.

Cerrillo, E. (1977): "Informe sobre las excavaciones realizadas en el yacimiento de 'El Cortinal de San Juan', Salvatierra de Tormes (Salamanca)", Noticiario Arqueológico Hispánico, 5, pp. 313-318

Díaz Martínez, P. C. y Martín Viso, I. (2011): “Una contabilidad esquiva: las pizarras numerales visigodas y el caso del Cortinal de San Juan (Salvatierra de Tormes, España)". En Díaz Martínez, P. C. y Martín Viso, I. (eds.): Entre el impuesto y la renta. Problemas de la fiscalidad tardoantigua y altomedieval. Bari, pp. 221-250.

Dorronsoro, C. (1991): "Suelos". En Gómez Gutiérrez, J. M (coord.): El libro de las dehesas salmantinas. Valladolid: JCyL, pp. 71-124.

García y Bellido, A. (1966): "Sobre la estela funeraria discoide de Valdenebro (Valladolid)". En IX Congreso Nacional de Arqueología. Valladolid, pp. 395-396.

Gómez-Moreno, M. (1967): Catálogo monumental de España. Provincia de Salamanca. Madrid: Ministerio de Educación y Ciencia.

Kajanto, I. (1965): The Latin Cognomina. Helsinki-Helsingfors: Keskurkirjapaino.

HüBner, E. (1869): Corpus Inscriptiorum Latinarum II. Inscriptiones Hispaniae Latinae. Berlin.

Maluquer de Motes, J. (1956): Carta Arqueológica de España. Salamanca. Salamanca: Diput. Prov. de Salamanca.

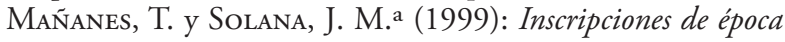
romana de la provincia de Valladolid. Valladolid: Univ. de Valladolid.

Morán, C. (1919): Investigaciones acerca de Arqueología y Prehistoria de la región salmantina. Salamanca: edic. Calatrava.

Morán, C. (1933): "De arqueología salmantina”, Boletín de la Real Academia de la Historia, 102, pp. 389-398.

Morán, C. ([1946]; 2000, 2. ${ }^{\text {a }}$ ed.): Reseña histórico artística de la provincia de Salamanca. Salamanca: Diput. Prov. de Salamanca.

PALOL, P. (1964): "La primera inscripción romana hallada en la provincia de Valladolid", Boletín del Seminario de Estudios de Arte y Arqueología, 30, pp. 307-311.

Rivero, C. M. a (1931): Museo Arqueológico Nacional. Adquisiciones en 1930. Madrid.

Rivero, C. M. ${ }^{\text {a }}$ (1933): El lapidario del Museo Arqueológico de Madrid. Valladolid.

VELÁzQUEz SORIANO, I. (1989): Las pizarras visigodas: edición, crítica y estudio. Serie vi Antigüedad y Cristianismo: Monografías históricas sobre la antigüedad tardía. Murcia.

VelázQuez Soriano, I. (2004): Las pizarras visigodas. Entre el latin y su disgregación. La lengua hablada en Hispania, siglos VI-VIII. Burgos: Instituto de la Lengua Castellano y Leonés.

Vives, J. (1970): Inscripciones latinas de la España Romana. Barcelona: UB-CSIC. 